\title{
Space conditions during a month of a sequence of six $M>6.8$ earthquakes ending with the tsunami of 26 December 2004
}

\author{
G. Anagnostopoulos and A. Papandreou \\ Demokritos University of Thrace, Space Research Laboratory, 67100 Xanthi, Greece \\ Correspondence to: G. Anagnostopoulos (ganagno@ee.duth.gr)
}

Received: 16 November 2010 - Revised: 30 June 2011 - Accepted: 11 July 2011 - Published: 21 May 2012

\begin{abstract}
This paper examines space and seismological data for the time period about one month before the giant Sumatra-Andaman strong (9.3) earthquake (EQ). The combination of seismological and space data reveals some interesting features for this time period: (1) six successive high speed solar wind streams obviously triggering a sudden increase of geomagnetic activity were all followed by strong to giant $(M>6.8) \mathrm{EQs}$, (2) the 6 strong EQs present certain spatial-temporal constraints, with the epicentre of the EQs occuring at the edges of the Pacific Plate (the SumatraAndaman EQ occurred at the end of this series of EQs, eastward of the first one), in a clockwise direction, (3) the EQs occurred after a sudden increase of geomagnetic activity, as inferred from the $3 \mathrm{~h}-K_{p}$ index, following a quiet geomagnetic period and (4) the time delay of the $M>6.2$ earthquakes (in the broad area examined) from the last maximum sudden $K_{p}$ increase was on average $\sim 1.5$ days. These findings from the study of the Earth's space environment during the month preceding the Sumatra-Andaman giant (9.3) EQ provide new information for a possible better understanding of the Sun-magnetosphere-lithosphere coupling.
\end{abstract}

\section{Introduction}

The presence of the Sun controls the planet Earth and the life on it to a highest degree. In particular, scientific progress during the space era has greatly improved our knowledge of the Sun and its emissions, and of the solar influence on the 3-D interplanetary space and the planetary magnetospheres. Therefore, several studies in the last years have been devoted to the investigation of the relations between solar activity and changes in the status of the magnetosphere-ionosphere- technological systems (Baker, 2005; Marhavilas, 2008; Lanzerotti, 2010), seismic activity (Sobolev et al., 2001; Gousheva et al., 2003), and human life and health (Halberg et al., 2000; Lowell and Davis, 2008).
In addition, over the last two decades, many researchers have been greatly interested in developing scientific tools for advancing space weather and EQ prediction (Hayakawa and Molchanov, 2002; Baker, 2005; Molchanov and Hayakawa, 2008; Pulinets and Boyarchuk, 2004). Space weather prediction research has been accepted as the main priority in space physics and there exists a general consensus on the possibility of some prediction (Li et al., 2001), whereas EQ prediction research, despite the increasing evidence for the existence of EQ precursory signals, is still a controversial issue.

In the following in this section, we review some of the studies dealing with the possible influence of the Sun on seismic activity. For instance, Khain and Khalilov (2008) presented statistical results which suggest that the spectra of $M>=7 \mathrm{EQs}$ and of Wolf numbers of sunspots display the same main harmonic, at $T \approx 10-11 \mathrm{yr}$, that is about the solar cycle period. Gousheva et al. (2003) in their statistical study found two maxima in the global yearly number of EQs in the 11-yr sunspot cycle: one maximum coinciding with the solar sunspot cycle maximum (correlated with the strong solar activity) and another maximum observed on the descending phase of the solar cycle (correlated with the solar wind streams incident on the magnetosphere in such a period).

Since the magnetosphere has been assumed to be a mediator in the Sun-seismic relationship, specific studies have been carried out to investigate the correlation between magnetospheric and seismic activity. For instance, Sobolev et al. (2001) and Zakrzhevskaya and Sobolev (2002) studied two sub-catalogs with seismic events in the areas of Kazakhstan and Kyrgyzstan (Mikhailova, 1990) preceding and following magnetic storms, and they found that the number of EQs occurring after storms increases in some areas and decreases in others, but that the tendency toward an increase of the number of EQs after the storm characterizes the region as a whole. A possible interpretation of this fact is that the compensation of the storm affects time intervals. These authors 
applied parametric and non-parametric criteria and inferred that the storm effect on seismicity was non-accidental, with a probability of $99.9 \%$. They also came to the conclusion that the seismic response to a magnetic storm lasts for 10 days, with a maximum effect between 2 to 7 days after sudden commencement.

Bakhmutov et al. (2007) presented an example of correlation between geomagnetic field disturbances and earthquakes on a specific seismic zone in Vrancea, in the South Carpathians. They studied more than 200 EQs during the period 1988-1996, and they found that $90 \%$ of seismic shocks recorded in the Vrancea zone were associated with mid-latitude manifestation of the near midnight polar substorms. These substorms manifested themselves both against the quiet geomagnetic field background and during magnetic storms. The authors noted that "When there are no substorms we have no records of deep-foci EQs in Vrancea at all". They also reported that definite morphological signs in the geomagnetic variation spectrum precede seismic activity. Their statistical results also suggest that the energy class of the EQ is related to the amplitude of the $\mathrm{H}$-component changes (during near-midnight polar substorm manifestation).

Odinstov et al. (2007) examined the solar wind speed increase, a particular cause of (sub)storms, and checked its possible impact on seismic activity. Their statistics showed that the maximum of the EQ number directly correlates with a sudden increase in the solar wind speed. Furthermore, a more specific study of solar activity influence on seismic activity has been conducted by Gousheva et al. (2003), who also found a relation between the solar wind speed hitting the Earth's magnetosphere and seismic activity. Gousheva et al. (2003) noted that although many authors have studied the role of extraterrestrial factors in terrestrial seismicity, the problem remains controversial. There exists increasing evidence for the influence of solar activity upon seismic activity, but we know that there are geomagnetic (sub)storms which are not accompanied by seismic activity, and EQs which are not related to (sub)storms. Despite the statistical evidence provided for the influence of the solar wind streams on seismic activity, the issue still remains controversial in the scientific community.

In this paper we examine the seismic activity compared with the space environment status for the time period of about one month before the deadly tsunami of 26 December 2004. Our analysis reveals some interesting relationships between the space variations and seismic activity during the time period examined: (1) all 6 of 6 successive solar wind speed increases were followed by one (or more) $M>6.8$ EQs, after $\sim 1.5$ days. (2) The series of successive strong EQs during the period examined present a spatial and temporal relation, with the epicentre of all strong EQs before the Sumatra EQ being located at the edges of the Pacific Plate, and the EQs occuring in a clockwise direction around the Pacific Plate (the Sumatra-Andaman EQ occurred at the end of this series of EQs, eastward of the first one). We
Table 1. Place, date/time, magnitude, Longitude, Latitude of $M>6.8$ earthquakes for the time period starting one month before the giant Sumatra-Andaman earthquake until the end of the year 2004, as given in the list of "Significant Earthquakes" of the US Geophysical Survey site.

\begin{tabular}{lccccc}
\hline No & Date & Time (UT) & Magn. & Long. & Lat. \\
\hline 1 & $26 \mathrm{Nov}$ & $02: 25: 03$ & 7.1 & $135.346^{\circ} \mathrm{E}$ & $3.6^{\circ} \mathrm{S}$ \\
2 & $28 \mathrm{Nov}$ & $18: 32: 13$ & 7.0 & $145.056^{\circ} \mathrm{E}$ & $43.0^{\circ} \mathrm{N}$ \\
3 & $6 \mathrm{Dec}$ & $14: 15: 11$ & 6.8 & $145.200^{\circ} \mathrm{E}$ & $42.9^{\circ} \mathrm{N}$ \\
4 & $14 \mathrm{Dec}$ & $23: 20: 13$ & 6.8 & $81.348^{\circ} \mathrm{W}$ & $19.0^{\circ} \mathrm{N}$ \\
5 & $23 \mathrm{Dec}$ & $14: 59: 03$ & 8.1 & $160.365^{\circ} \mathrm{E}$ & $50.1^{\circ} \mathrm{S}$ \\
6 & $26 \mathrm{Dec}$ & $00: 58: 53$ & 9.3 & $95.854^{\circ} \mathrm{E}$ & $3.3^{\circ} \mathrm{N}$ \\
\hline
\end{tabular}

hypothesize that these special temporal-spatial constraints of EQs most probably reflect an underlying geological process that provided the appropriate conditions for the sudden geomagnetic activity to act as a triggering cause provoking the 6 great to giant successive EQs between 26 November-26 December 2004.

\section{Observations}

The terrible tsunami following the Sumatra-Andaman EQ on 26 December 2004 has been studied from many points of view. Here we study the history of seismic activity during the month preceding the giant EQ taking into account the conditions of the space environment. In our study, we use and compare values of the geomagnetic $K_{p}$ index and the solar wind speed $V$ retrieved from the NOAA Space Weather Prediction Center (http://www.swpc.noaa.gov/) and the USGS EQ lists (http://earthquake.usgs.gov). The relevant time period from 23 November 2004 to 31 December 2004 is examined. We have plotted these data in composite figures in an attempt to search for any qualitative correlations between parameters of space weather, geomagnetic status and earthquakes. In addition, we present some quantitative results in order to provide a preliminary quantification of these correlations.

In Table 1 we provide information (place, date/time, magnitude, longitude, latitude) for the time period starting one month before the giant Sumatra-Andaman EQ until the end of the year 2004, as given in the list of "Significant Earthquakes" of the US Geophysical Survey site (http://earthquake.usgs.gov/earthquakes/eqinthenews/). The catalog includes $6 \mathrm{EQs}$ with magnitude $M>=6.8$. The six EQs occurred in the following regions: (\#1) Papua Islands, Indonesia, on 14 November $(M=7.1)$, (\#2) Near Hokaido, Japan region, on 28 November, $(M=7.0)$, (\#3) Near Hokaido, on 23 December 2004, $(M=6.8)$, (\#4) 


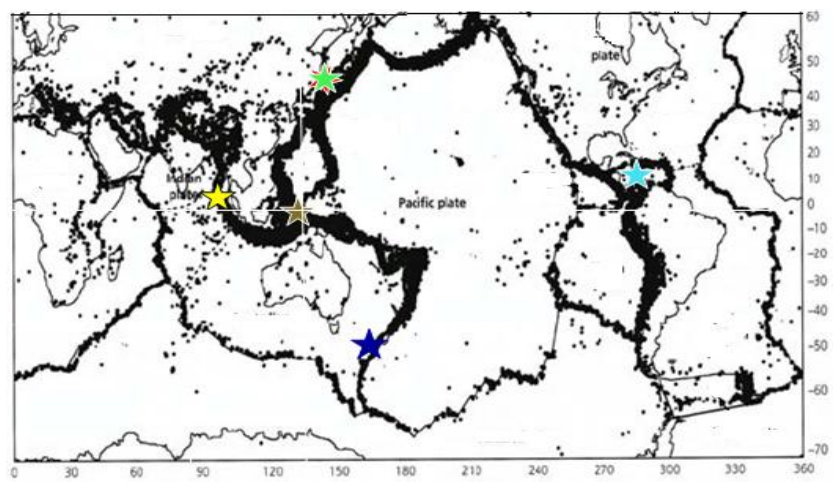

Fig. 1. Map with the position of the epicentre of the 6 great $(M=6.8)$ earthquakes in the month preceding the Sumatra 2004 earthquake. The earthquakes were located at the edge of Pacific Plate.

Cayman Islands, on 24 December, $(M=6.8)$, (\#5) North of Macquarie Islands, on 6 December, $(M=8.1)$ and $(\# 6)$ Off the west coast of northern Sumatra, on 26 December $(M=9.3)$; in the following, each one of the $6 \mathrm{EQs}$ earthquakes will be called by its number in the above list; for instance the EQ occurring in Papua Islands will be called EQ \#1, whereas that in Sumatra will be called EQ \#6. Figure 1 displays a map with the position of the epicentres of the 6 EQs.

From a comparison of the data given in Table 1 and Fig. 1, some spatial and temporal constraints are evident:

1. the epicentres of all six EQs (are not dispersed, but all of them) are located in a certain region of the globe,

2. the epicentres of the first five EQs are located at the edges of the Pacific Plate, with the epicentre of the EQ \#6 (Sumatra), the last one in this sequence of the six EQs, lying "close" to the Pacific Plate, eastward of the first one (EQ \#1),

3. all five EQs, \# 1-2-3-4-5, were occurred in a clockwise direction around the Pacific Plate, with the EQ \#6 (Sumatra-Andaman EQ) occurring after and close to the EQ \#5 occurring in the southwest edge of this Plate,

4. the two greatest EQs in the sequence of the six $>6.8$ EQs occurred at the end: EQ \#5 $(M=8.1)$ occurred relatively close and only before $\sim 3$ days from EQ \# 6 (Sumatra-Andaman, $M=9.3$ ).

The spatial-temporal ordering of the six greatest EQs which occurred within a month in a unique geological structure of the world most probably suggests that certain common underlying geological processes were in progress during the month preceding the Sumatra-Andaman EQ; this special underlying geological process might be responsible for the unexpected strength of this EQ (Stein and Okal, 2007).
Table 2. The six earthquakes of Fig. 1: magnitude, date of occurrence, the days of the monotonic $K_{p}$ increase before each of the 6 earthquakes, the values of the increasing $K_{p}$ index before each earthquake, the corresponding index increase over its value of the previous day $\Delta K_{p} / \Delta t$ (\#/day), and the total increase $\Sigma \Delta K_{p}$ during the period (days) of $K_{p}$ index monotonic increase.

\begin{tabular}{|c|c|c|c|c|c|c|}
\hline No & Magnitude & $\begin{array}{l}\text { Date } \\
\text { (EQ) }\end{array}$ & Date & $K_{p}$ & $\begin{array}{r}\Delta K_{p} / \Delta t \\
(\# / \text { day })\end{array}$ & $\Sigma \Delta K_{p}$ \\
\hline \multirow[t]{4}{*}{1} & 7.1 & $26 \mathrm{Nov}$ & & & & \\
\hline & & & $25 \mathrm{Nov}$ & 27 & 10 & 15 \\
\hline & & & $24 \mathrm{Nov}$ & 17 & 5 & \\
\hline & & & $23 \mathrm{Nov}$ & 12 & & \\
\hline \multirow[t]{2}{*}{2} & 7.0 & $28 \mathrm{Nov}$ & $28 \mathrm{Nov}$ & 22 & 3 & 3 \\
\hline & & & $27 \mathrm{Nov}$ & 19 & & \\
\hline \multirow[t]{3}{*}{3} & 6.8 & $6 \mathrm{Dec}$ & $6 \mathrm{Dec}$ & 28 & 13 & 28 \\
\hline & & & $5 \mathrm{Dec}$ & 15 & 15 & \\
\hline & & & $4 \mathrm{Dec}$ & 0 & & \\
\hline \multirow[t]{4}{*}{4} & 6.8 & $14 \mathrm{Dec}$ & & & & 12 \\
\hline & & & $12 \mathrm{Dec}$ & 31 & 10 & 12 \\
\hline & & & $11 \mathrm{Dec}$ & 21 & 2 & \\
\hline & & & $10 \mathrm{Dec}$ & 19 & & \\
\hline \multirow[t]{5}{*}{5} & 8.1 & $23 \mathrm{Dec}$ & & & & 21 \\
\hline & & & $22 \mathrm{Dec}$ & 26 & 6 & \\
\hline & & & $21 \mathrm{Dec}$ & 20 & 12 & \\
\hline & & & $20 \mathrm{Dec}$ & 8 & 3 & \\
\hline & & & 19 Dec & 5 & & \\
\hline \multirow[t]{3}{*}{6} & 9.3 & $26 \mathrm{Dec}$ & & & & 16 \\
\hline & & & $25 \mathrm{Dec}$ & 22 & 16 & \\
\hline & & & 24 Dec & 6 & & \\
\hline
\end{tabular}

In Fig. 2 we display the magnitude of the six EQs indicated by normal bars at the particular time of EQ occurrence (top panel), along with the daily numbers of the geomagnetic activity index $K_{p}$ (middle panel) and the daily variation of the same index $\Delta K_{p}$ (bottom panel); the six significant (>6.8) EQs (Table 1) are numbered as \#1-6 above the panel. From Fig. 2 we can clearly see that 5 of 6 EQs, namely EQs $\# 1,3,4,5$, and 6 , occurred after days of enhanced geomagnetic activity as suggested by both the monotonic increasing values of $K_{p}$ (Panel b) and the high positive values of the daily rate $\Delta K_{p}$ (Panel c).

In Fig. 3 we extend our investigation to the space environment outside the magnetosphere: we introduce the concept of the plasma emitting from the Sun which is known as "solar wind". The solar wind moves away from the Sun with an average speed of $350 \mathrm{~km} \mathrm{~s}^{-1}$, extends up to the Termination Shock of the heliosphere and influences the Earth's magnetosphere (as well as the other planetary magnetospheres). In particular, in Fig. 3 we show the geomagnetic index $K_{p}$ (top panel), along with the magnitude of the interplanetary magnetic field (IMF) $B$ (middle panel) and the value of the solar wind speed $V$ (bottom panel); the measurements of $B$ and $V$ have been made by the ACE spacecraft. The time profiles of $K_{p}, B$ and $V$ suggest correlation of Earthquakes \#2, 3, 4, 

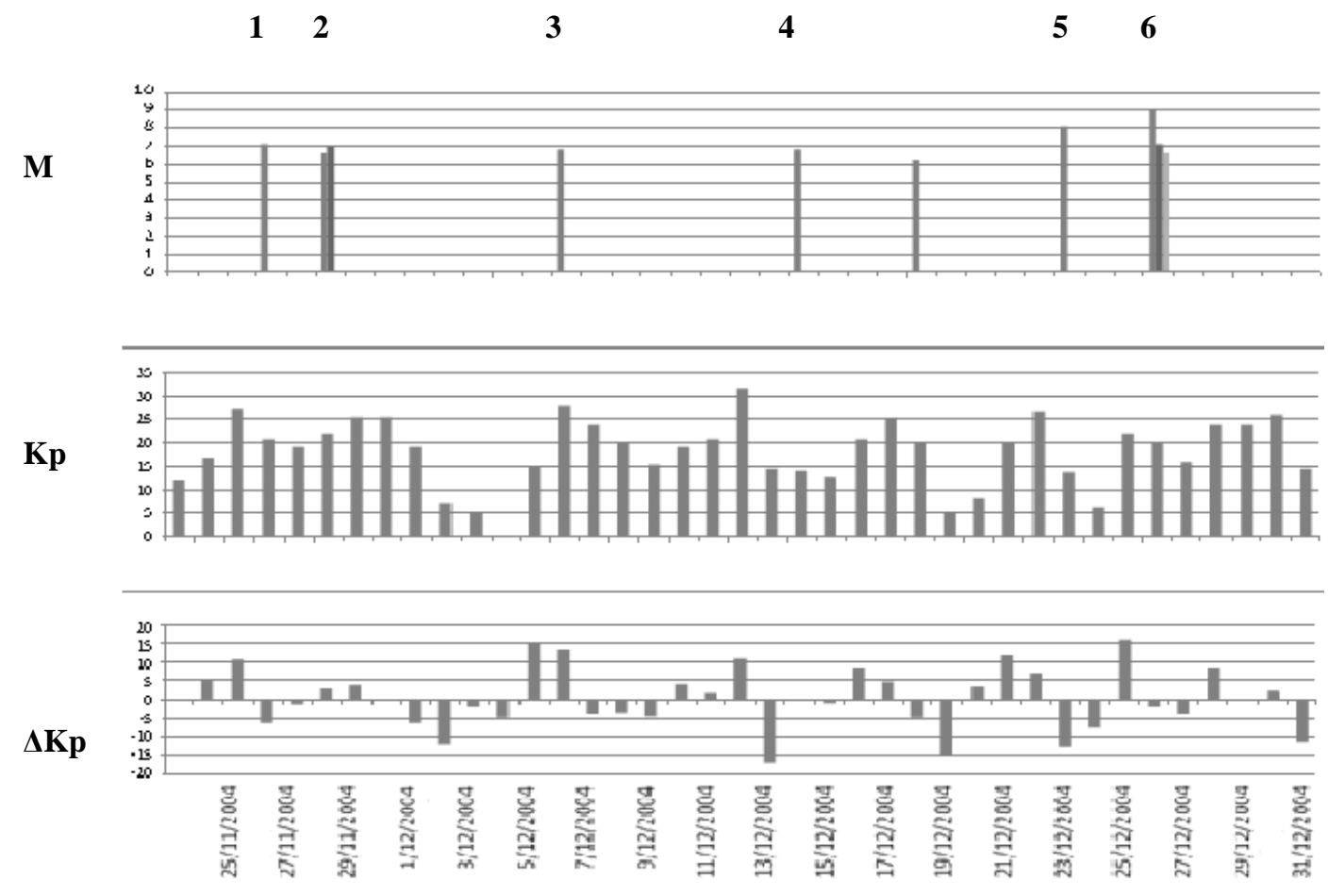

Fig. 2. The figure displays the magnitude of the six earthquakes (the top panel) along with the daily numbers of the geomagnetic activity index $K_{p}$ (middle panel) and the daily variation the same index $\Delta K_{p}$ (bottom panel); the six significant (>6.8) earthquakes shown in Table 1 are numbered as \#1-6 above the panel.

5 and 6, not only with the $K_{p}$ increases as already seen in Fig. 2, but also with increases in magnetic field $B$ and solar wind speed $V$. These times of simultaneous magnetic field $B$ and solar wind speed $V$ increases indicate the times when the leading edge of the high speed solar wind streams arrived in the environment of the magnetosphere. The coincidence of the arrival of these solar wind streams with $K_{p}$ increases is a well known process in space physics, where solar wind streams incident on the Earth's magnetosphere can trigger enhanced geomagnetic activity.

In Fig. 4 we further examine the relation of the solar wind speed increases with the enhanced geomagnetic activity. In this figure we compare the daily values of the geomagnetic index $K_{p}$ (top panel) and the daily average solar wind speed $V$ (third panel from the top), along with the corresponding daily rates of variations $\Delta K_{p}$ (second panel from the top) and $\Delta V$ (bottom panel). The normal arrows in the figure were drawn to indicate the enhanced geomagnetic activity following the solar wind speed increases for all 6 significant earthquakes (\#1-6).

In Table 2 we elaborate the level of geomagnetic activity and its change preceding the 6 EQs (Figs. 1, 2). The columns of the table (left to the right) show the numbering of EQs (\#1-6), their magnitude, the date of their occurrence, the days of the monotonic $K_{p}$ increase preceding each
EQ, the values of the increasing $K_{p}$ index in those days, the corresponding index increase over its value of the previous day $\Delta K_{p}$ (\#/day), and the total increase $\Sigma \Delta K_{p}$ during the period (days) of monotonic increase of the $K_{p}$ index. (The behavior of the geomagnetic $K_{p}$ index shown in the last columns of Table 2 reflects, in numbers, the $K_{p}$ bar profile of Figs. 2, 4).

Important to note from Table 2 are the following:

1. in 4 of 6 cases (EQ \#1, 4, 5 and 6), the EQs occurred after the day of maximum $K_{p}$ value,

2. the two strongest EQs are characterized by preceding days of very low geomagnetic activity (Min- $K_{p}=5$ before EQ \#5 and Min- $K_{p}=6$ before EQ \#6; compared to $K_{p}=12,19$ and 19 before EQs \#1, 2, and 4),

3. the same EQs are also characterized by high maximum daily increase (Max- $\Delta K_{p}=12$ before EQ \#5 and Max$\Delta K_{p}=16$ before $\mathrm{EQ} \# 6$; compared to $\Delta K_{p}=10,3$ and 10 before EQs \#1, 2, and 4),

4. EQ \#3 which presented extreme Min- $K_{p}(=0)$ and Max- $\Delta K_{p}(=15)$ does not correspond to the strongest $\mathrm{EQ}$, as we would expect in agreement with the previous Points \# 2 and 3, but it should be noted that this EQ 


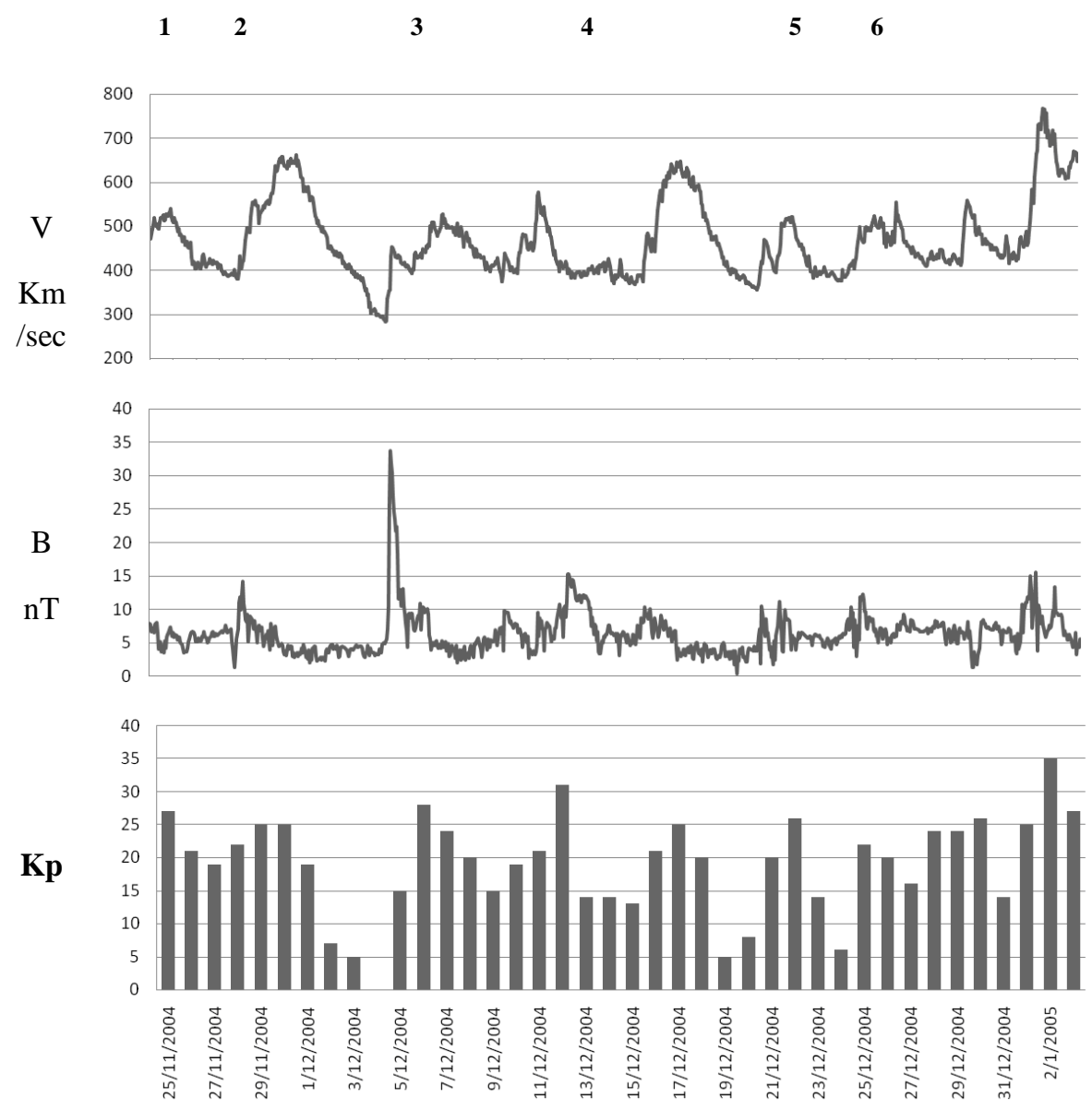

Fig. 3. The geomagnetic index $K_{p}$ (top panel) along with the magnitude of the interplanetary magnetic field (IMF) $B$ (middle panel) and the value of the solar wind speed $V$ (bottom panel); the time profiles of $K_{p}, B$ and $V$ suggest a correlation of Earthquakes \#2, 3, 4, 5 and 6, not only with the $K_{p}$ increases but also with increases in magnetic field $B$ and solar wind speed $V$.

occurred on the same day as EQ \#2 with an epicenter at almost the same place (Hokkaido, Japan), and

5. the EQs \# 6, 5 and 3 show a similar behavior for the total $\Sigma \Delta K_{p}$ (last column) as that seen for the daily Min- $K_{p}$ and Max- $\Delta K_{p} / \Delta t$ values (Points \#2-4).

The above characteristics extracted from Table 2 for the month before the Sumatra EQ have been found to exist in several other cases, and we have been working on statistics to estimate the appearance of such characteristics. Here we should recall that the six EQs examined follow a certain pattern concerning their correlation with $V, K_{p}$, Min- $K_{p}$, Max$\Delta K_{p}$ and $\Sigma \Delta K_{p}$ and that this is most probably due to the common spatial-temporal constraints during the period examined.

In order to study the time relationship between the geomagnetic increase activity and the EQ occurrence time for the 6 cases examined here in more detail, we display the magnitude of the great EQs at the certain time of occurrence (top panel) in Fig. 5, along with the numbers of the 3-h geomagnetic activity index $K_{p}$ (bottom panel). Since in
Fig. 2, a distinct solar wind stream with gradually increasing speed on days 16 and 17 November 2004 was accompanied by enhanced geomagnetic activity and was followed by an EQ with $M=6.2$ on day 18 November 2004 not included in the USGS list of significant EQs, we wanted to include it in Fig. 5 and study the possible relations of all EQs in the examined region with magnitudes $M>=6.2$ with space physics parameters. The results coming from Fig. 5 and tabulated in Table 3 are surprising. First, from Fig. 5 we can observe that each of the EQs marked now as \#1, 2-, 2, 3, 4, *, 5, 6 are preceded by enhanced $\left(\Delta K_{p} \geq 2\right)$ values of the 3 -h $K_{p}$ index (indicated by arrows in the bottom panel). Second, an evaluation of the time difference $\Delta t=T_{\mathrm{EQ}}-T_{M}$ between the EQ occurrence time and the time of the last maximum $K_{p}$ value before the corresponding $\mathrm{EQ}$ suggests that $\Delta t$ varies in a small range of time, between $30-42 \mathrm{~h}$. The average value of the time difference $\Delta t=T_{\mathrm{EQ}}-T_{M}$ is estimated to be $\Delta t=34.9 \approx 35 \mathrm{~h}$, that is about 1.5 days after the sudden increase of the geomagnetic activity.

In order to check the statistical significance of the time difference $\left(\Delta T=T_{\mathrm{EQ}}-T_{M} \approx 35 \mathrm{~h}\right)$ found between the $\mathrm{EQ}$ 


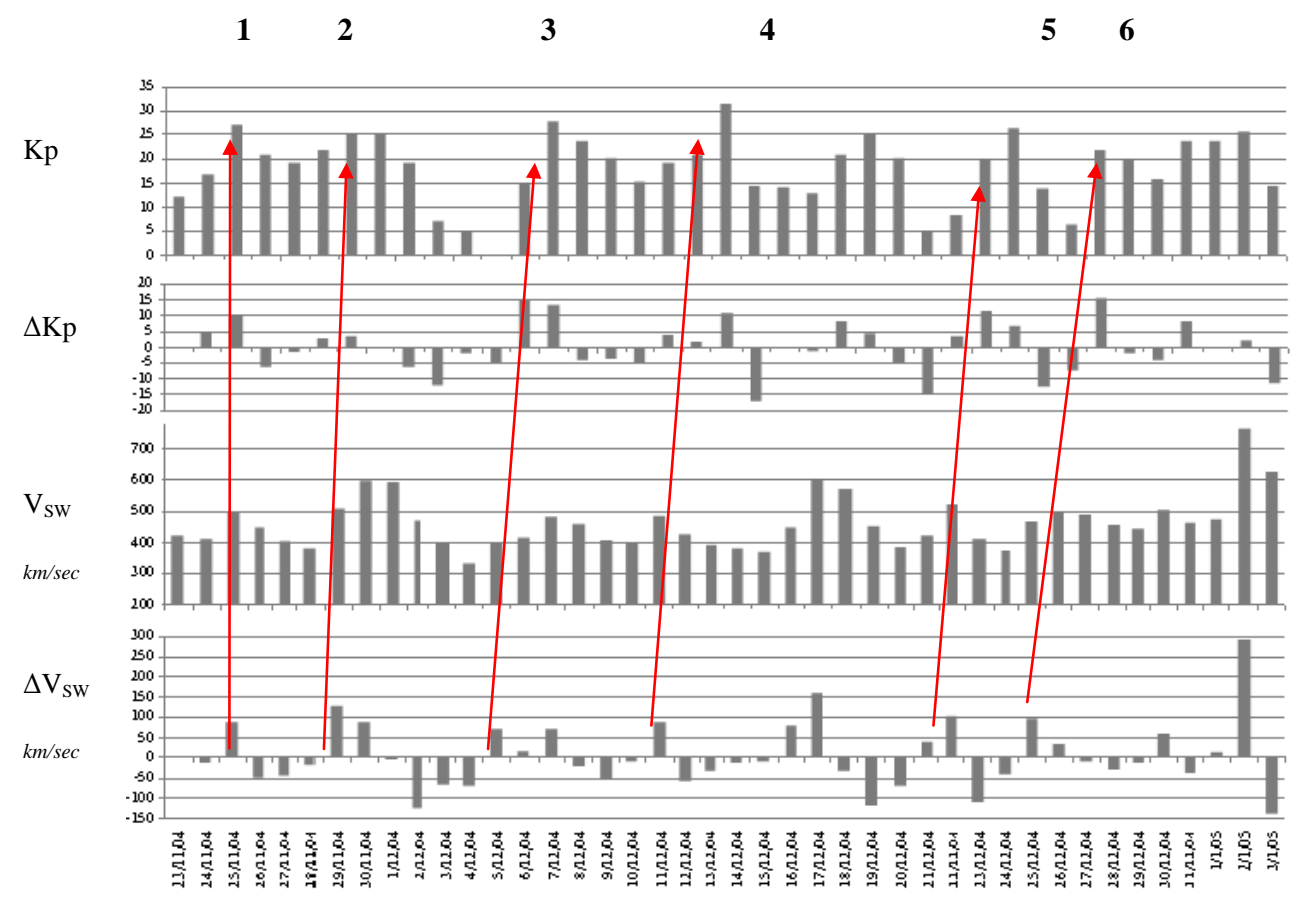

Fig. 4. Daily values of the geomagnetic index $K_{p}$ (top panel) and the daily average solar wind speed $V$ (third panel from the top) along with the corresponding daily rates of variations $\Delta K_{p}$ (middle panel) and $\Delta V$ (bottom panel). The normal arrows indicate the enhanced geomagnetic activity following solar wind speed increases for all 6 significant earthquakes (\#1-6).
$12-2$
3
$4 * 5$
56
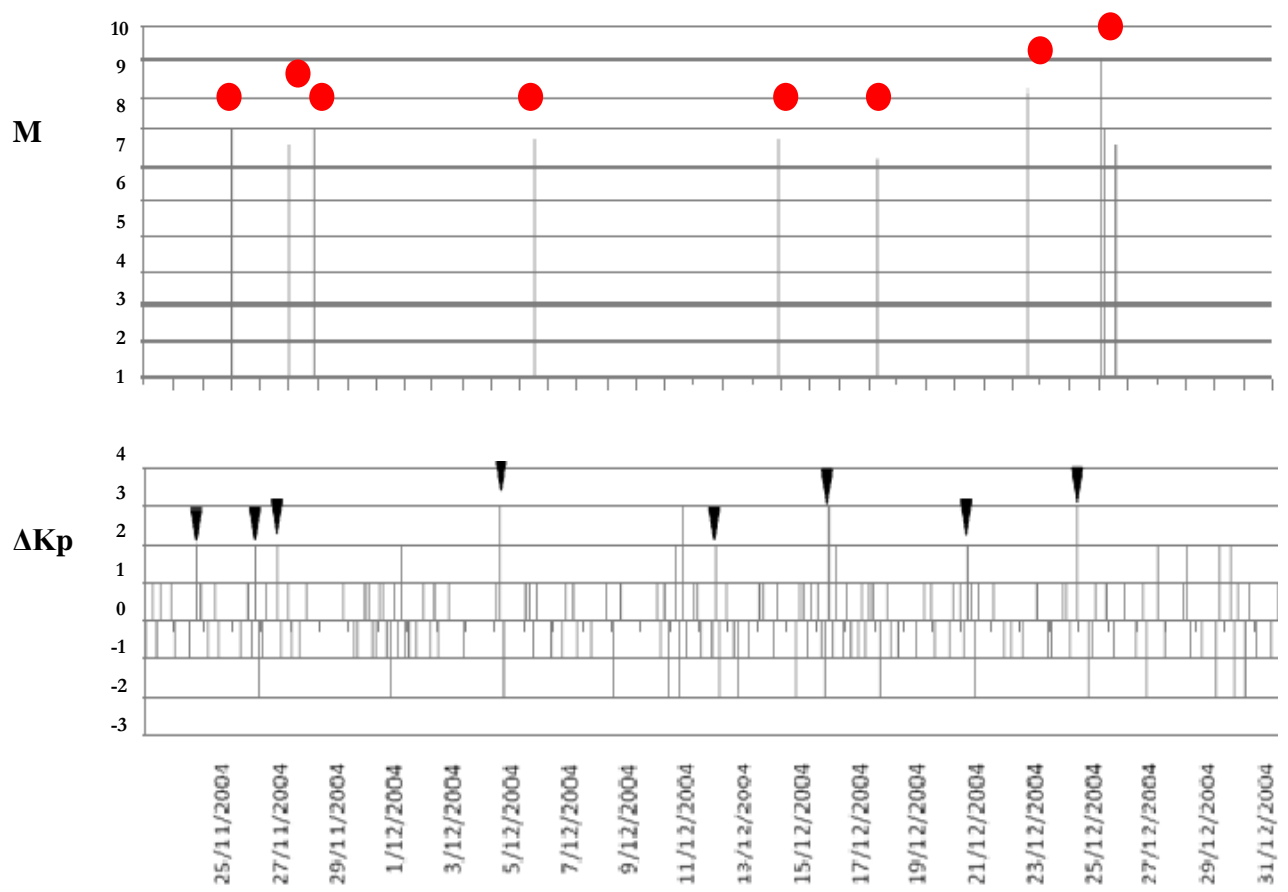

Fig. 5. The magnitude of the great $(M>6.2)$ earthquakes (top panel) along with the numbers of the geomagnetic activity 3 -h $K_{p}$ index are presented (bottom panel). The time difference between earthquake occurrence and the time of the last maximum $K_{p}$ value before the corresponding EQ suggests that $\Delta t$ varies in a small range of time, between $30-42 \mathrm{~h}$. The average value of the time difference is estimated to be about 1.5 days. 
Table 3. Time difference $\Delta t=T_{\mathrm{EQ}}-T_{M}$ between the earthquake occurrence time and the time of the last maximum $K_{p}$ value before the corresponding EQ. The average value of the time difference $\Delta t=T_{\mathrm{EQ}}-T_{M}$ is evaluated to be $\Delta t \approx 35 \mathrm{~h}$, that is about one and a half days after the sudden increase of $K_{p}$ index.

\begin{tabular}{lccc}
\hline No & Date & Magnitude & $T_{\mathrm{EQ}}-T_{M}(\mathrm{~h})$ \\
\hline 1 & 26 Nov 2004 & 7.1 & 36 \\
$2-$ & 28 Nov 2004 & 6.6 & 33 \\
2 & 28 Nov 2004 & 7.0 & 30 \\
3 & 6 Dec 2004 & 6.8 & 33 \\
4 & 14 Dec 2004 & 6.8 & 33 \\
$*$ & 18 Dec 2004 & 6.2 & 36 \\
5 & 23 Dec 2004 & 8.1 & 42 \\
6 & 26 Dec 2004 & 9.3 & 36 \\
\hline
\end{tabular}

Mean 34.9

occurrence time and the time of the last maximum $K_{p}$ value before the corresponding EQ during the month of a sequence of 8 earthquakes ending with the tsunami of 26 December 2004, we perform two statistical tests. First, we perform the Sign Test. We test the hypothesis that " $35 \mathrm{~h}$ is the median of the eight $\Delta T$ values of Table 3". For this reason we compare each of the eight value $\Delta T$ with the "median" $<\Delta T>=35$ and we note the sign of the difference $\Delta T-<\Delta T>$. The comparison gives the following series of signs:,,,,,,,+----+++ , where "-" corresponds to values $\Delta T<\langle\Delta T\rangle$ and "+" to $\Delta T\rangle\langle\Delta T\rangle$. The smaller value of + and - signs is 4 ; this number is higher than the critical number provided by the appropriate Table for the Sign Test (Dixon and Mood, 1946) for a total number of 8 signs, at a significant level of $P=0.01$ for a two-tailed test. Therefore, according to the Sign Test the null hypothesis is not rejected.

For further testing the statistical significance of the correlation between the EQ occurrence time $T_{\mathrm{EQ}}$ and the time $T_{M}$ of the last maximum $K_{p}$ value before the corresponding $\mathrm{EQ}$, we evaluate the correlation coefficient for the 8 pairs of times $T_{\mathrm{EQ}}$ and $T_{M}$ of Table 3. In Fig. 6 we plot the times $T_{\mathrm{EQ}}$ versus $T_{M}$. Since our statistical sample is not normally distributed, we evaluate the nonparametric Spearman rank correlation coefficient $r_{\mathrm{s}}$. We test the null hypothesis that "there is a significant linear correlation between the values $T_{\mathrm{EQ}}$ and $T_{M}$ of Table 3" The Spearman rank correlation coefficient is found to be $r_{\mathrm{s}}=0.797 \approx 0.8$ and by using the appropriate Table (Johnson and Leone, 1964) we see that $\mathrm{r}_{\mathrm{s}}$ is lower than the critical value 0.833 for $P=0.02$, that means a Spearman rank correlation coefficient $r_{\mathrm{s}} \approx 0.8$ statistically significant at the level of $P=0.02$ (coincidence chance $2 \%$ ). A line in Fig. 6 has been drawn that indicates the linear correlation between the values $T_{\mathrm{EQ}}$ and $T_{M}$; the horizontal distance from the diagonal corresponds to the median time delay of the time of the $\mathrm{EQ}\left(T_{\mathrm{EQ}}\right)$ from the time of the last sudden increase in geomagnetic index $K_{p}$ before the corresponding EQ $\left(T_{M}\right)$.

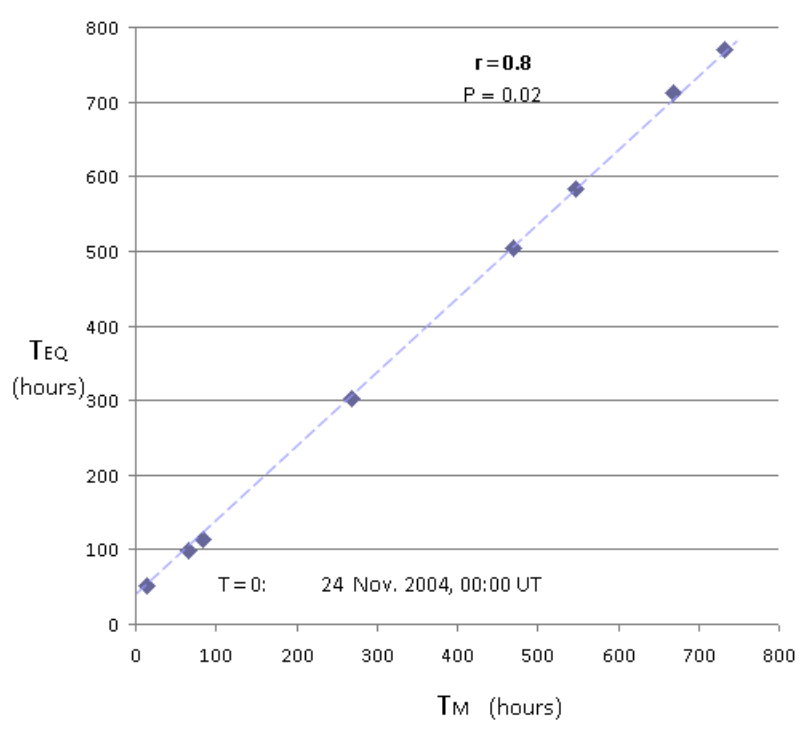

Fig. 6. The time of the earthquake occurrence $T_{\mathrm{EQ}}$ versus the time of the last maximum $K_{p}$ value $T_{M}$ before the corresponding EQ (see in the text). A strong correlation $(r=0.8)$ was found between the eight pairs of the values $T_{\mathrm{EQ}}$ and $T_{M}$ considered.

\section{Discussion}

The Sun-earth relationship is a central point in many research directions nowadays and some Sun-Earth relationships have already been confirmed and have been incorporated into the corps of several scientific areas. In particular, the space science community has concentrated its interest on so-called "space weather" in order to predict geomagnetic and ionospheric disturbances, which have a significant impact on electrical power systems, telecommunications, oil pipelines, spacecraft and aircraft electronics, astronauts safety, etc. (Baker, 2005; Marhavilas, 2008; Lanzerotti, 2010). Scientific research has also provided some evidence on the relation of solar-magnetospheric activity with EQs.

However, the Sun-interplanetary space-magnetosphereionosphere-atmosphere-lithosphere chain is a complicated, open, dynamic, nonlinear system of a complex of processes, with high unpredictability. In this context, the seismic phenomena of the Earth should be considered as a part of the whole Sun-Earth system. The relation between seismicity and the Sun-Earth chain processes has been considered as ambiguous, and for this reason the contribution of various solar impact processes on Earth's lithosphere and their association with the seismic energy release needs much work.

In this paper we investigated an extended time period of 35 days (23 November 2004 to 28 December 2004), which includes the deadly Sumatra-Andaman $(M=9.3)$ EQ of 26 December 2004. This interesting time interval studied from many points of view by several authors reveals surprising results concerning the status of space parameter changes 
before 6 strong EQs: the most striking feature is that all 6 successive strong $(M>6.8)$ earthquakes were preceded by enhanced geomagnetic activity (Fig. 2) within $\sim 1.5$ days (Table 3). The existence of such a permanent feature and in a sequence of $6 \mathrm{EQs}$, most probably suggests that some special geological conditions (Stein and Okal, 2007) during the period examined allowed such an ordering. Indeed, the seismological data support such a hypothesis since we observed that the series of successive EQs present a spatial and temporal ordering (Fig. 1 and relative discussion in the text/Sect. 2). This special temporal-spatial earthquake ordering most probably reflects a common underlying geological process, in metastable state, which provided the conditions for the high speed solar wind streams/rapidly increasing geomagnetic activity to act in some way as the EQ triggering cause.

Besides the above-discussed evidence for the solar wind high speed streams/geomagnetic activity-related seismic activity relationship in the time period examined, our data analysis provides significant information which is probably related to the nature of the triggering mechanism. Our analysis suggests that the strong EQs occurred at times of sudden increase geomagnetic activity following a geomagnetic quiet period. The high value $K_{p}$ index time rate variation suggests a sudden geomagnetic disturbance, and therefore, intense induced electric phenomena close to Earth.

Sobolev et al. (2001) and Zakrzhevskaya and Sobolev (2001) have already argued that if the crust in the area of a forthcoming EQ is in metastable state, it becomes sensitive to trigger-like effects. They speculated that the electrical energy supplied to the earth during a storm is converted into mechanical energy via piezoelectric, electrokinetic or other mechanoelectric effects and increases mechanical stresses. They noted that a comparison of the energy supplied by magnetic storms and that released by EQs suggests that a magnetic storm can only act as a triggering mechanism, not as the main cause of the seismic activity. In addition, Duma and Ruzhin (2003) demonstrated a probabilistic relation of ionospheric $\mathrm{Sq}$ currents with seismic activity.

Some authors suggested other extraterrestrial processes as triggering mechanisms of seismic activity: solar and lunar tides (Jakubkova and Pick, 1987), solar proton fluxes (Velinov, 1975), and earthward movement of the magnetopause (Makarova and Shirochkov, 1999).

Kormiltsev et al. (2002) hypothesized that magnetic storms induce electro-osmotic fluid flows which causes EQ triggering due to the influence of anomalous porous pressure.

Sytinskii (1997) argued that the EQ triggering mechanism is the solar- induced change in atmospheric circulation expressed in the large scale reorganization of |baric fields, while Prikryl et al. (2003) provided evidence that gravity waves are generated by auroral electrojets caused by high speed solar wind MHD waves.
Tarasov et al. (1999) reported a probable triggering effect of impulsive electrical signals on seismicity based on a study of the number of EQs in Kyrgyzstan associated with electrical signals radiated by an MHD generator. They inferred that the number of EQs tends to increase 3-4 days after the electrical signal passage at distances of the order of a few hundred kilometres. Based on these results, Doda (2001) proposed that we can regard the electric currents induced in the Earth's crust by magnetic disturbances as the same result of the MHD generator impact.

\section{Conclusions}

This paper examines space and seismological data for the time period about one month before the giant SumatraAndaman giant (9.3) EQ followed by the tsunami that caused the death of hundreds of thousands people in a large part of our planet. The combination of seismological and space data reveals three striking results for this time period:

1. a series of successive six high speed solar wind streams/sudden increase of geomagnetic activity were all followed by strong to giant $(6.8<M<9.3)$ earthquakes within $\sim 1.5$ days after the incidence of the solar wind streams on the Earth's magnetosphere,

2. the series of successive strong EQs presents a spatial and temporal relation, with the epicentre of the EQs occuring in a clockwise direction at the edges of the Pacific Plate (the Sumatra-Andaman EQ occurred at the end of this series of EQs, eastward of the first one), and

3. the EQs occurred after a rapidly disturbed magnetosphere following a quiet geomagnetic period.

In conclusion, we infer that before the Sumatra-Andaman EQ, some special geological conditions in the Pacific Plate probably prepared the appropriate conditions for provoking very strong $\mathrm{EQs}$ within $\sim 1.5$ days after a sudden disturbance of a quiet magnetosphere.

The results presented in this study are in agreement with the findings of previous authors who found some statistical evidence for a relationship between geomagnetic and seismic activity (Sobolev et al., 2001; Zakrzhevskaya and Sobolev, 2002; Bakhmutov et al., 2007) and between solar wind speed increases and seismic activity (Gousheva et al., 2003; Odinstov al., 2007). However, we need further studies to check to what extent and under which additional conditions such relationships are possible, and to advance our understanding of Sun-magnetosphere-ionosphere-lithosphere coupling processes. 
Acknowledgements. The leading author thanks P. Antoniou for the preparation of some of the figures used in this paper, and interesting discussions on this paper. He also thanks I. Veselovsky, A. Rigas and P. Preka for helpful discussions and their comments on the manuscript. We also thank the three referees of this paper for their constructive comments.

Edited by: M. E. Contadakis

Reviewed by: P. F. Biagi and two other anonymous referees

\section{References}

Baker, N. D.: Introduction to Space Weather, Lecture Notes in Physics, 656, 993-1020, doi:10.1007/978-3-540-31534-6_1, 2005.

Bakhmutov, V., Sedova, F., and Mozgovaya, T.: Morphological Features in the Structure of Geomagnetic Variations in Relation to Earthquakes in Vrancea, Publ. Inst. Geophys. Pol. Acad. Sc., 43, 931-937, doi:10.1134/S1069351307110031, 2007.

Doda, L.: Geoseismic echo of solar storms, Air fleet, 3, 56-57, 2001.

Dixon, J. W. and Mood, A. M.: The Statistical Sign Test, J. Amer. Statist. Ass., 41, 557-566, 1946.

Duma, G. and Ruzhin, Y.: Diurnal changes of earthquake activity and geomagnetic Sq-variations, Nat. Hazards Earth Syst. Sci., 3, 171-177, doi:10.5194/nhess-3-171-2003, 2003.

Gousheva, M. N., Georgieva, K. Y., Kirov, B. B., and Atanasov, D.: On the Relation Between Solar Activity and Seismicity, RAST 2003, Proceed. International Conf. "Recent Advances in Space Technologies", Istanbul, Turkey, edited by: Kurnaz, S., Ince, F., and Onbaioglu, S., IEEE Catalog Number 03EX743. ISBN: 0-7803-8142-4, Library of Congress Catalog Card Number 2003109595, the Institute of Electrical and Electronics Engineers, Inc., 236 pp., 2003.

Halberg, F., Cornélissen, G., Otsuka, K., et al.: Cross-spectrally coherent $\sim 10.5$ - and 21-yr biological and physical cycles, magnetic storms and myocardial infractions, Neuroendocrinolgy Letters, ISSN 172-780X, 21, 233-258, 2000.

Hayakawa, M. and Molchanov, O. A. Editors, Seismo Electromagnetics: Lithosphere-Atmosphere-Ionosphere Coupling, TERRAPUB, Tokyo, 477 pp., 2002.

Jakubcova, J. and Pick, M.: Correlation between solar motion, earthquakes, and other geophysical phenomena, Ann. Geophys., 5, 135-141, 1987, http://www.ann-geophys.net/5/135/1987/.

Khain, V. E. and Kalilov, E. N.: About the possible influence of solar activity upon seismic and volcanic activities: long-term forecast, Science without borders, Trans. $f$ the International Academy of Science H\&E., SWB, 2070-0334, 3, 217-240, 2008.

Kormiltsev, V. V., Kostrov, N. P., Ratushnyak, A. N., and Shapiro, V. A.: The influence of electro-osmotic pressure generated by geomagnetic disturbances on the evolution of seismotectonic process, in: Electromagnetic Lithosphere-Atmosphere-Ionosphere Coupling, edited by: Nayakawa, M. and Molchanov, O. A., Terrapub, Tokyo, 203-207, 2002.
Lanzerotti, L. J.: Pipelines and Space Weather, Space Weather, 8, S05006, doi:10.1029/2010SW000594, 2010.

Li, X., Temerin, M., Baker, D. N., Reeves, G. D., and Larson, D.: Quantative prediction of radiation belt electrons at geostationary orbit based on solar wind measurements, Geophys. Res. Lett., 28, 1887-1890, doi:10.1029/2000GL012681, 2001.

Lowell, E. W. and Davis, G. E.: The light of life: Evidence that the sun modulates human lifespan, Med Hypotheses, 70, 501-507, doi:10.1016/j.mehy.2007.05.053, 2008.

Makarova, L. N. and Shirochkov, A. V.: On the connection between the Earth's magnetosphere magnetopause position and the earthquakes occurrence, Abstracts of XXVI General Assembly LJRSI, Toronto, Canada, 13-21, p. 755, 1999.

Marhavilas, P.: Space as natural laboratory of Electronics, J. Engin. Sci. and Tech. Review, 1, 9-18, 2008.

Mikhailova, N. N.: Catalog of earthquakes in the North Tien Shan and Adjacent Areas over the Period from 1975 through 1982: Parts 1 and 2, Alma-Ata: Nauka, 1990.

Molchanov, O. A. and Hayakawa, M.: Seismo Electromagnetics and Related Phenomena: History and latest results, TERRAPUB, Tokyo, 189 pp., 2008.

Odintsov, S. D., Ivanov-Klodonyi, G. S., and Gergieva, K.: Solar activity and Global seismicity on Earth, Bull. Russ. Aca. Sci., Physics, 71(4), 593-597, 2007.

Prikryl, P., Muldrew, D. B., and Sofko, G. J.: High-speed solar wind, auroral electrojets and atmospheric gravity waves: a link to the Earth's atmosphere, Proceedings International Solar Cycle Studies Symposium 2003 "Solar Variability as an Input to the Earth Environment”, Tatranska Lomnica, Slovakia, ESA SP, 23-28, 2003.

Pulinets, S. and Boyarchuk, K.: Ionospheric Precursors of earthquakes, Springer, ISBN3-540-20839-9, 2004.

Sobolev, G. A., Zakrzhevskaya, N. A., and Kharin, E. P.: On the relation between seismicity and magnetic storms, Phys. Solid Earth, Russian Acad. Sc., 11, 62-72, 2001.

Stein, S. and Okal, E. A.: Ultralong period seismic study of the December 2004 Indian Ocean earthquake and implications for regional tectonics and the subduction process, Bull. Seismol. Soc. Am., 97, S279-S295, doi:10.1785/0120050617, 2007.

Sytinskii, A. D.: Influence of interplanetary disturbances on the seismicity and atmosphere of the Earth, Geomagn. Aeron., 37, 138-143, 1997.

Tarasov, N. T., Tarasova, N. V., Avagimov, A. A., and Zeigarnik, V. A.: The effect of high-power electromagnetic pulses on the seismicity of the Central Asia and Kazakhstan, Volcan. Seismol., Russian Acad. Sc. 4/5, 152-160, 1999.

Velinov, P.: The effect of solar activity on geophysical processes, Bulg. Geofiz. Spis., 1, 51-77, 1975.

Zakrzhevskaya, N. A. and Sobolev, G. A.: On the seismicity effect of magnetic storms, Phys. Solid Earth, Russian Acad. Sc., 4, 315,2002 . 